\title{
Signed Laplacian Deep Learning with Adversarial Augmentation for Improved Mammography Diagnosis
}

\author{
Heyi Li ${ }^{1 \star}$, Dongdong Chen ${ }^{1 *}$, William H. Nailon ${ }^{2}$, \\ Mike E. Davies ${ }^{1}$, and David I. Laurenson ${ }^{1}$ \\ 1 Institute for Digital Communications, University of Edinburgh, Edinburgh, UK \\ \{Heyi.Li,d.chen, dave.laurenson, mike.davies\}@ed.ac.uk \\ 2 Oncology Physics Department, Edinburgh Cancer Centre, Western General \\ Hospital, Edinburgh bill.nailon@luht.scot.nhs.uk
}

\begin{abstract}
Computer-aided breast cancer diagnosis in mammography is limited by inadequate data and the similarity between benign and cancerous masses. To address this, we propose a signed graph regularized deep neural network with adversarial augmentation, named DiAGNET. Firstly, we use adversarial learning to generate positive and negative mass-contained mammograms for each mass class. After that, a signed similarity graph is built upon the expanded data to further highlight the discrimination. Finally, a deep convolutional neural network is trained by jointly optimizing the signed graph regularization and classification loss. Experiments show that the DiAGNET framework outperforms the state-of-the-art in breast mass diagnosis in mammography.
\end{abstract}

Keywords: Deep learning · Mammography Diagnosis · Adversarial learning · Graph regularization

\section{Introduction}

Breast cancer is one of the most frequently diagnosed mortal diseases for women all over the world 6. Mammography is extensively applied and computer-aided diagnosis systems (CADs) are often employed as a second reader. Leveraging the recent success of deep neural networks on representation learning, deep learning

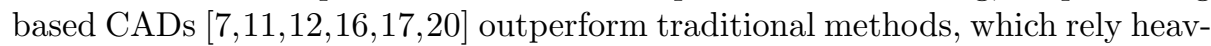
ily on handcrafted features. However, two major challenges in mammographic CADs remain (1): limited access to well annotated data 7 and (2): the similarity between benign and cancerous masses. To alleviate the impact of inadequate

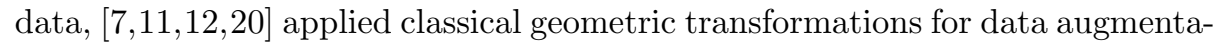
tion (e.g. flips, rotations, random crops etc), and more recently, 16 17] generated synthetic images on the manifold of real mammograms using adversarial learning 9], which enjoys a powerful ability to learn the unknown underlying distribution. Unfortunately, the following questions remain unanswered: What kind of data

* These authors contribute equally to this work. 
augmentation is most helpful for CADs in mammography? How can we alleviate the impact of the similarity between data, i.e., how can we maximize the margin between manifolds with a small difference?

In this paper, we propose a new deep learning framework that improves mammography diagnosis as follows. Firstly, we propose an adversarial data augmentation strategy, in which both positive and negative samples of specific classes are generated in an unsupervised manner, in order to make more distinct boundaries between different classes. After that, we build a signed graph Laplacian over the augmented data to quantitatively capture the geometric structure of data. Finally, we train a deep neural network by jointly optimizing the graph regularization and classification loss, by which the intra-class difference is minimized, and more importantly, the inter-class manifold margin is maximized in the deep representation space. Extensive experiments show that the proposed DiagNeT outperforms the state-of-the-art of breast masses diagnosis in mammography.

\section{Preliminary}

\subsection{Adversarial learning}

Adversarial learning is a technique that attempts to fool models through malicious input 10 and has achieved impressive results in representation learning. The key idea of the success of a Generative Adversarial Network (GAN) is to force the output of the generator to be indistinguishable from the real input 9 . Adversarial training is particularly powerful for image generation, and for learning unknown and complicated distributions from the training data. In this paper, we propose to use adversarial learning to generate off-distribution instances along with on-distribution instances in order to enlarge the medical image data.

\subsection{Manifold Learning}

In real applications, data typically reside on a low-dimensional manifold embedded into a high-dimensional ambient space 15. Manifold learning is extensively explored because of its effectiveness for preserving the topological locality, which relies on the assumption that neighbors tend to have the same labels [3]. In this paper, we aim to incorporate graph embedding into a deep neural network as a regularizer in the latent space. In addition, local data manifold structure preservation within the hidden representations in deep neural networks offers the possibility of improving the performance of the classifier [4].

\section{Proposed Method}

In this section, we formally introduce the details of DIAGNET, which is composed of three steps as shown in Fig.1. (1) adversarial augmentation, (2) a signed graph Laplacian built upon the augmented data and (3) joint optimization of the classifier loss and signed graph regularizer. We first define the notation applied 


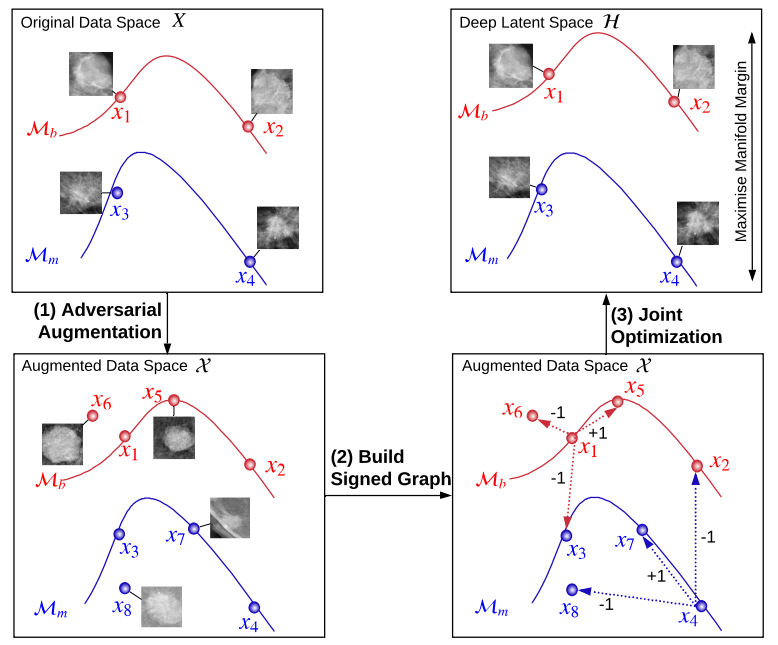

(a)

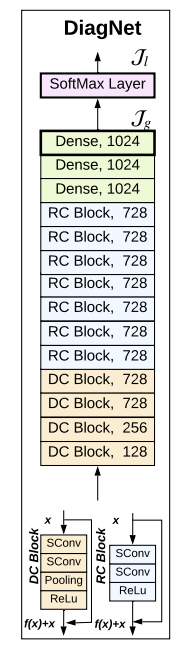

(b)

Fig. 1: The proposed DiagNet for Breast Mass Diagnosis. (a) the framework of the proposed algorithm, which consists of three steps. $\left\{x_{1}, x_{2}\right\}$ and $\left\{x_{3}, x_{4}\right\}$ are samples on benign manifold $\mathcal{M}_{b}$ and malignant manifold $\mathcal{M}_{m}$ respectively. In the first step, i.e. adversarial data augmentation, positive neighbors $\left\{x_{5}, x_{7}\right\}$ and negative neighbors $\left\{x_{6}, x_{8}\right\}$ are generated with (1) and (2) respectively. Then a signed graph is built upon both original and augmented samples as (3). Finally, a joint loss (6) is optimized in the deep latent space, maximizing data manifold margin. (b) The utilized deep network architecture. "DC block" represents a down-sampling convolutional block, "RC block" is a residual convolutional block, and "SConv" is separable convolutions.

throughout the paper. Let $\{X, Y\}=\left\{\boldsymbol{x}_{i}, y_{i}\right\}_{i=1}^{n}$ be the $n$ mammograms with corresponding labels, where $\boldsymbol{x}_{i} \in \mathbb{R}^{H \times W}$ is an image sample and $y_{i} \in\left\{y_{c}\right\}_{c=1}^{C}$ is the class label. Let $\left\{X_{c}, y_{c}\right\}$ denote the $c$-th class data.

\subsection{Adversarial Augmentation}

As also mentioned in section 1, inadequate data and the similarity between benign and cancerous masses $\overline{7}$ are two main reasons causing high false positives in mammographic CADs. Recently, 1, 16, 17 employed GANs to create new instances. Even though they generated on-distribution samples that are not separable by discriminators, they ignored the importance of distinguishable but similar instances, which tend to improve the discriminative ability. To overcome this shortcoming, as shown in Fig 1(a), we propose to use adversarial learning to generate more instances of both positive neighbors (i.e. instances on the manifold, e.g. $x_{5}$ and $x_{7}$ ) and negative neighbors (i.e. instances off the manifold, e.g. 
$x_{6}$ and $\left.x_{8}\right)$. Here, there are defined two manifolds: $\mathcal{M}_{b}$ for benign images and $\mathcal{M}_{m}$ for malignant images.

In particular, inspired by [19], we generate neighboring instances one by one for a certain data class $\left\{\overline{X_{c}}, y_{c}\right\}, c=1,2, \cdots, C$, where $C=2$ in this paper. Specifically, both positive and negative neighbors are generated based on the noise corrupted seed points (a number of randomly selected samples in $X_{c}$ ) and they are both close to the original data points. In particular, the positive neighbors $X_{c}^{+}$are the generated samples that cannot be separated from $X_{c}$ by a discriminator, while the negative neighbors $X_{c}^{-}$are the ones that can be separated. Finally, the expanded dataset for class $c$ is of the form $\mathcal{X}_{c}=$ $\left\{X_{c} \cup X_{c}^{+} \cup X_{c}^{-}\right\}$, and the whole dataset is $\mathcal{X}=\bigcup_{c} \mathcal{X}_{c}$.

Let $\boldsymbol{x}$ be a desired new sample and $P\left(\boldsymbol{x} ; X_{c}, X_{c}^{+}\right)$be the probability that $\boldsymbol{x}$ is classified as class $c$ by a discriminator trained on $\left\{X_{c}, X_{c}^{+}\right\}$. Similarly $P\left(\boldsymbol{x} ; X_{c}, X_{c}^{-}\right)$corresponds to a discriminator trained on $\left\{X_{c}, X_{c}^{-}\right\}$. Note that $X_{c}^{+}$and $X_{c}^{-}$are initialized as empty. In this paper, we trained two SVM classifiers as the discriminators and the corresponding output probability is obtained with logistic sigmoid of the output signed distance. Accordingly, a set of neighboring instances $\left\{\boldsymbol{x}_{t}\right\}_{t=1}^{T}$ of $X_{c}$ are iteratively generated. In each iteration $t$, the discriminator is learned and the weights are updated. After $T$ iterations of training, we select one desired positive neighbor $\boldsymbol{x}$ :

$$
\underset{\boldsymbol{x}}{\arg \max } P\left(\boldsymbol{x} ; X_{c}, X_{c}^{+} \cup\left\{\boldsymbol{x}_{t}\right\}_{t=1}^{T}\right)-\gamma \max \left\{0, r_{1}-\min _{\boldsymbol{x}_{i} \in X_{c}^{+}} d\left(\boldsymbol{x}, \boldsymbol{x}_{i}\right)\right\},
$$

where $d(\cdot)$ is a distance measure, $\gamma$ weights the distance regularization, forcing generated points to be different with a minimum distance $r_{1}$. Similarly, we select one desired negative neighbor $\boldsymbol{x}$, with an added distance restriction to force new points to be scattered close to $X_{c}$ :

$$
\begin{aligned}
\underset{\boldsymbol{x}}{\arg \min } P\left(\boldsymbol{x} ; X_{c}, X_{c}^{-} \cup\left\{\boldsymbol{x}_{t}\right\}_{t=1}^{T}\right) & +\gamma \max \left\{0, r_{2}-\min _{\boldsymbol{x}_{j} \in X_{c}^{-}} d\left(\boldsymbol{x}, \boldsymbol{x}_{j}\right)\right\} \\
& +\gamma \max \left\{0, \min _{\boldsymbol{x}_{i} \in X_{c}} d\left(\boldsymbol{x}, \boldsymbol{x}_{i}\right)-r_{3}\right\},
\end{aligned}
$$

where the distance regularization forces generated points to acquire a minimum distance $r_{2}$ and maximum distance $r_{3}$.

\subsection{Signed graph Laplacian regularizer}

Graph embedding trained with distributional context can boost performance in various pattern recognition tasks. In this paper, we aim to incorporate the signed graph Laplacian regularizer [2] to learn a discriminative datum representation $\mathcal{H}(\mathcal{X})$ by a deep neural network, where discriminative here means that the intraclass data manifold structure is preserved in the latent space and the intermanifold (slightly different) margins are maximized.

Using the supervision of the adversarial augmentation in section 3.1, we build a signed graph upon the expanded data $\mathcal{X}$. Given $\mathcal{X}_{c}=\left\{X_{c}, X_{c}^{+}, X_{c}^{-}\right\}$for class 
$c$, and all other classes data $\mathcal{X}_{-c}=\bigcup_{t=1, \cdots, C: t \neq c}\left\{X_{t}, X_{t}^{+}, X_{t}^{-}\right\}$, for $\forall \boldsymbol{x}_{i} \in \mathcal{X}_{c}$, the corresponding elements in the signed graph is built as follows:

$$
\phi_{i j}= \begin{cases}+1, & \boldsymbol{x}_{j} \in\left\{X_{c} \cup X_{c}^{+}\right\}_{i}^{n^{+}}, \\ -1, & \boldsymbol{x}_{j} \in\left\{X_{-c} \cup \mathcal{X}_{c}^{-}\right\}_{i}^{n^{-}},\end{cases}
$$

where the $\{\cdot\}_{i}^{n^{+}}\left(\{\cdot\}_{i}^{n^{-}}\right)$denotes the corresponding $n^{+}\left(n^{-}\right)$nearest neighborhood of $x_{i}$ to approximate the locality of the manifold.

Then, we compute the structure preservation in the deep representation space (directly behind the softmax layer as shown in Fig $1(\mathrm{~b})) \mathcal{H}=\left\{h\left(\boldsymbol{x}_{i}\right)\right\}_{i=1}^{N}$, where $N=|\mathcal{X}|$. The signed graph Laplacian regularizer is defined as following:

$$
\mathcal{J}_{g}(\mathcal{X}, \Phi)=\sum_{i, j} \begin{cases}\phi_{i j} \cdot \operatorname{dist}\left(h\left(\boldsymbol{x}_{i}\right), h\left(\boldsymbol{x}_{j}\right)\right), & \text { if } \phi_{i j}>0 \\ \max \left(0, m+\phi_{i j} \cdot \operatorname{dist}\left(h\left(\boldsymbol{x}_{i}\right), h\left(\boldsymbol{x}_{j}\right)\right)\right), & \text { if } \phi_{i j}<0,\end{cases}
$$

where $\operatorname{dist}(\cdot)$ is a distance metric for the dissimilarity between $h\left(\boldsymbol{x}_{i}\right)$ and $h\left(\boldsymbol{x}_{j}\right)$. It encourages similar examples to be close, and those that are dissimilar to have a distance of at least $\mathrm{m}$ each other, where $m>0$ is a margin.

Note that instead of calculating the manifold embedding by solving an eigenvalue decomposition, we learn the embedding $\mathcal{H}$ by a deep neural network. Specifically, inspired by the depth-wise separable convolutions [5] that are extensively employed to learn mappings with a series of factoring filters, we build stacks of depth-wise separable convolutions with similar topological architecture to that in [5] to learn such deep representations (Fig 1(b)].

Therefore, by minimizing (4), it is expected that if two connected nodes $\boldsymbol{x}_{i}$ and $\boldsymbol{x}_{j}$ are from the same class (i.e. $\phi_{i j}$ is positive), $h\left(\boldsymbol{x}_{i}\right)$ and $h\left(\boldsymbol{x}_{j}\right)$ are also close to each other, and vice versa. Benefiting from such learned discriminativity, we train a simple softmax classifier to predict the class label, i.e.,

$$
\mathcal{J}_{l}=-\frac{1}{N} \sum_{i=1}^{N} \sum_{c=1}^{C} \delta_{c}\left(y_{i}\right) \log P\left(y_{i} \mid \boldsymbol{x}_{i} ; \boldsymbol{\theta}\right),
$$

where $\delta_{c}\left(y_{i}\right)=1$ when $y_{i}=c$, and 0 otherwise; $\boldsymbol{\theta}$ is the parameter set of the neural network.

Finally, by incorporating the signed Laplacian regularizer (4) and the classification loss (5), the total objective of DIAGNET is accordingly defined as:

$$
\mathcal{J}=\mathcal{J}_{l}+\lambda \mathcal{J}_{g}
$$

where $\lambda \geq 0$ is the regularization trade-off parameter which controls the smoothness of hidden representations.

\section{Experiments}

\subsection{Datasets and ROIs selection}

The DiagNet is evaluated on the most frequently used full-field digital mammographic dataset, INbreast 13. 107 mass contained mammograms are divided 
into a training and a test set containing $80 \%$ and $20 \%$ of the images respectively. As for ROIs selection, rectangular mass-contained boxes are selected with proportional padding (1.6 times) upon original ROI bounding boxes. The selected ROIs are augmented with flips and further adversarially augmented by $40 \%$ more ( $20 \%$ positive neighbors and $20 \%$ negative neighbors).

\subsection{Implementation Details}

We first solve the proposed adversarial augmentation in (1) and (2) by the derivative-free optimization approach RACOS algorithm [18]. The distance measure $d(\cdot)$ in (1) and (2) is set to be the angular cosine distance because of its superior discriminative information [14. Let $\rho=\min _{\boldsymbol{x}_{i}, \boldsymbol{x}_{j} \in \mathcal{X}_{c}} d\left(\boldsymbol{x}_{i}, \boldsymbol{x}_{j}\right)$, then we set the radius parameters $r_{1}, r_{2}=\rho$, and $r_{3}=3 \times \rho$ for $\mathcal{X}_{c}$. Further $T=200$ and $\gamma$ is $10^{-2}$.

Secondly, the signed graph is built upon augmented data $\mathcal{X}$. For each graph node, $n^{+}$and $n^{-}$in (3) are optimally chosen as 1 and 4 respectively using grid search. In addition, the metric $\operatorname{dist}(\cdot)$ in (4) is also the angular cosine distance and $m$ is 1 .

Finally, the deep neural network is built with stacks of $3 \times 3$ kernel-sized separable convolutional layers. The first three blocks are equipped with increasing feature maps $(128,256,728)$ and decreasing spatial squared size $(224,112,56)$, and the consecutive seven blocks keep the same feature map with size 28. After global averaging and three fully connected layers of 1024 neurons, a softmax layer is padded for label prediction. Dropout layers with $50 \%$ dropout rate and weight decay with $l_{2}$ norm rate $10^{-4}$ are used to prevent over-fitting. Residual skips are added in order to solve the gradient diverging and vanishing problems. The regularization parameter $\lambda$ in (6) is optimally chosen as 1 .

\subsection{Results and analysis}

Adversarial Augmentation: To examine the quality of generated images by the proposed adversarial augmentation strategy, we carry out the experiment on the INbreast dataset. Fig 2 visually shows the augmented examples. It can be seen that, for either mass type, the generated positive and negative neighbors are both similar to the original data, but the negative neighbors are more different.

Compare to the state-of-art: We validate DIAGNET's performance with accuracy and AUC (area under the ROC curve) scores. Table 1 compares the state-of-art algorithms, in which 11 is re-implemented and the results of the remaining ones are taken from the original papers. It shows that, the DiAGNET has achieved the state-of-art with mean accuracy $93.4 \%$ and AUC score 0.95. When compared with the second best algorithm [16], the DiagNeT's AUC score is significantly higher with experiments on the whole dataset without any pre-processing, post-processing or transfer learning. In addition, empirical observations show that our model is robust to noise and geometric transforms, and these results are omitted due to the space limitation. 


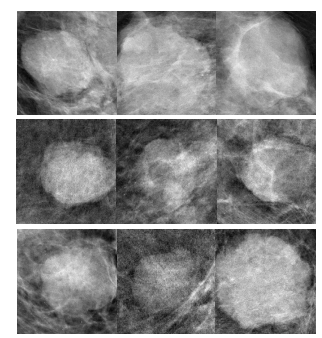

(a) Benign Masses

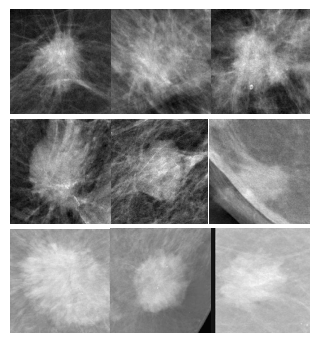

(b) Malignant Masses

Fig. 2: Generated mammogram examples by proposed adversarial augmentation strategy. The masses in the first row of both (a) and (b) are original data, the second and the third row are generated positive and negative neighbors, respectively.

Table 1: Breast Mass Diagnosis performance comparisons of the proposed DIAGNET and relative state-of-the art methods on INbreast test set.

\begin{tabular}{l|c|c|c}
\hline Methodology & End-to-end & Accuracy & AUC \\
\hline (2012) Domingues et. al 8 & $\times$ & $89 \%$ & N/A \\
(2016) Dhungel et. al 7 8 & $\checkmark$ & $91 \%$ & 0.76 \\
(2017) Zhu et. al 20 & $\checkmark$ & $90 \%$ & 0.89 \\
(2018) Shams et. al 16] & $\checkmark$ & $93 \%$ & 0.92 \\
(2019) Li et. al 11] & $\checkmark$ & $88 \%$ & 0.92 \\
\hline proposed DiAGNET & $\checkmark$ & $\mathbf{9 3 . 4} \pm 1.9 \%$ & $\mathbf{0 . 9 5 0} \pm 0.02$ \\
\hline
\end{tabular}

Importance of Signed Graph Laplacian regularizer: Determining the optimal values of hyper-parameter is a big challenge in deep learning. To explore DIAGNET's performance with different signed graph configurations, the values of $n^{+}$and $n^{-}$are first grid searched with fixed regularization parameter $\lambda=1$, as shown in Fig 3(a). The best performance occurs when $n^{+}=1$ and $n^{-}=4$, which increases at least by $8 \%$ the accuracy rate and by $12 \%$ the AUC score compared to the baseline (no graph regularization, $n^{+}, n^{-}=0$ ). This confirms the effectiveness of using the signed graph regularization. In addition, results show that the DIAGNET achieves good performance only when both $n^{+}$and $n^{-}$ are considered in the corresponding singed graph construction. Fig 3 shows the performances with various values of $\lambda$, where the best result occurs at $\lambda=1$.

\section{Conclusions}

In this paper, we proposed a DiAGNET for improved mammogram image analysis. By integrating the signed graph regularizer and the adversarial sampling augmentation, DIAGNET works in a simple but effective way to learn discriminative features. Extensive experiments show that our method outperforms stateof-the-art on breast mass diagnosis in mammography. 


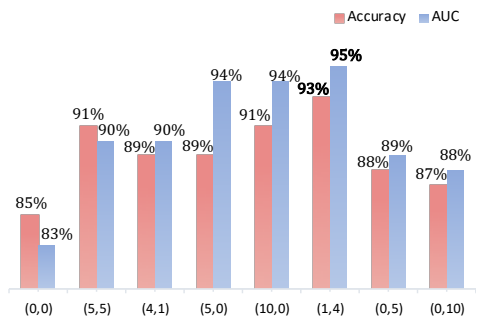

(a) Configurations of $\left(n^{+}, n^{-}\right)$

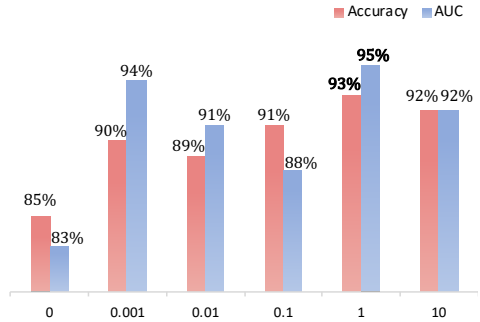

(b) Configurations of $\lambda$

Fig. 3: Performance of DiagNeT on INBreast with varying parameters. Classification accuracy and AUC score versus (a) different $n^{+}$positive neighbors and $n^{-}$negative neighbors and (b) various regularizer parameter $\lambda$.

\section{References}

1. Antoniou, A., Storkey, A., Edwards, H.: Data augmentation generative adversarial networks. arXiv preprint arXiv:1711.04340 (2017)

2. Chen, D., Lv, J., Davies, M.E.: Learning discriminative representation with signed Laplacian restricted Boltzmann machine. arXiv preprint arXiv:1808.09389 (2018)

3. Chen, D., Lv, J., Yi, Z.: Unsupervised multi-manifold clustering by learning deep representation. In: Workshops at the 31th AAAI conference on artificial intelligence (AAAI). pp. 385-391 (2017)

4. Chen, D., Lv, J., Yi, Z.: Graph regularized restricted boltzmann machine. IEEE Transactions on Neural Networks and Learning Systems 29(6), 2651-2659 (2018)

5. Chollet, F.: Xception: Deep learning with depthwise separable convolutions. In: Proceedings of the IEEE conference on computer vision and pattern recognition. pp. 1251-1258 (2017)

6. DeSantis, C., Ma, J., Bryan, L., Jemal, A.: Breast cancer statistics, 2013. CA: a cancer journal for clinicians 64(1), 52-62 (2014)

7. Dhungel, N., Carneiro, G., Bradley, A.P.: The automated learning of deep features for breast mass classification from mammograms. In: International Conference on Medical Image Computing and Computer-Assisted Intervention. pp. 106114. Springer (2016)

8. Domingues, I., Sales, E., Cardoso, J., Pereira, W.: INbreast-database masses characterization. XXIII CBEB (2012)

9. Goodfellow, I., Pouget-Abadie, J., Mirza, M., Xu, B., Warde-Farley, D., Ozair, S., Courville, A., Bengio, Y.: Generative adversarial nets. In: Advances in neural information processing systems. pp. 2672-2680 (2014)

10. Kurakin, A., Goodfellow, I.J., Bengio, S.: Adversarial machine learning at scale (2017)

11. Li, H., Chen, D., Nailon, W.H., Davies, M.E., Laurenson, D.: A deep dual-path network for improved mammogram image processing. International Conference on Acoustics, Speech and Signal Processing (2019)

12. Li, H., Chen, D., Nailon, W.H., Davies, M.E., Laurenson, D.: Improved breast mass segmentation in mammograms with conditional residual U-Net. In: Image Analysis for Moving Organ, Breast, and Thoracic Images, pp. 81-89. Springer (2018) 
13. Moreira, I.C., Amaral, I., Domingues, I., Cardoso, A., Cardoso, M.J., Cardoso, J.S.: INbreast: toward a full-field digital mammographic database. Academic radiology 19(2), 236-248 (2012)

14. Nair, V., Hinton, G.E.: Rectified linear units improve restricted Boltzmann machines. In: Proceedings of the 27th international conference on machine learning (ICML-10). pp. 807-814 (2010)

15. Seung, H.S., Lee, D.D.: The manifold ways of perception. science 290(5500), 22682269 (2000)

16. Shams, S., Platania, R., Zhang, J., Kim, J., Park, S.J.: Deep generative breast cancer screening and diagnosis. In: International Conference on Medical Image Computing and Computer-Assisted Intervention. pp. 859-867. Springer (2018)

17. Wu, E., Wu, K., Cox, D., Lotter, W.: Conditional infilling GANs for data augmentation in mammogram classification. In: Image Analysis for Moving Organ, Breast, and Thoracic Images, pp. 98-106. Springer (2018)

18. Yu, Y., Qian, H., Hu, Y.Q.: Derivative-free optimization via classification. In: Thirtieth AAAI Conference on Artificial Intelligence (2016)

19. Yu, Y., Qu, W.Y., Li, N., Guo, Z.: Open-category classification by adversarial sample generation. International Joint Conference on Artificial Intelligence (2017)

20. Zhu, W., Lou, Q., Vang, Y.S., Xie, X.: Deep multi-instance networks with sparse label assignment for whole mammogram classification. In: International Conference on Medical Image Computing and Computer-Assisted Intervention. pp. 603-611. Springer (2017) 